\begin{tabular}{|l|l|l|l|}
\hline Eiszeitalter u. Gegenwart & $\mathbf{3 2}$ & $\begin{array}{c}1-12 \\
5 \mathrm{Abb}, 1 \mathrm{~T} \text { Taf. }\end{array}$ & Hannover 1982 \\
\hline
\end{tabular}

\title{
Die Packeisküste Japans
}

\author{
Ludwig Ellenberg \& Kazuomi Hirakawa *)
}

\begin{abstract}
Glacial feater, pack ice, coastal dune, wave, current, climate effect, thermal pollution.
\end{abstract} NE-Hokkaido

Kurzfassung: Die Küsten des nördlichen Hokkaidos gehören zu den äquatornächsten Bereichen, die in saisonalem Wechsel einer wellen- und strömungsdominierten Sommerformung (8-10 Monate) und einer packeis- und frostinduzierten Winterformung (2-4 Monate) unterliegen. Es werden für diesen Raum die typischen Prozesse der Sommer- und Wintermonate beschrieben. Im Sommer werden die Küsten wesentlich stärker geformt als im Winter und die durch Eispressung entstandenen Formen an den Stränden und Dünen überdauern nur in Relikten. Dennoch kann die Zone der saisonal vereisten Küsten klimageomorphologisch von den Küsten wärmerer Breiten abgegrenzt werden, da nur hier ein derart großer Kontrast zwischen Sommer- und Winterformung existiert. Diese Grenze ist allerdings ein breiter Saum und kein schmaler Ubergang, wie es für solche physisch geographischen Grenzen die Regel ist.

\section{[The Pack Ice Coast of Japan]}

The coasts of northern Hokkaido belong to the areas next to the Equator which are formed in seasonal change dominated by waves and currents during summer $(8-10$ months) and pack ice and frost action during winter $(2-4$ months). The typical processes of summer and winter of this area are being described in this study. The process of coastal development is much more intense during summer than during winter and the forms originated from ice-shove at the beaches and dunes are outlasting only in relics. Nevertheless the zone of the coasts glaciated seasonally can be separated from coasts of warmer latitudes in view of the climatic geomorphology, because only in this case such a big contrast is existing between the development during summer and that in winter. This boundary indeed is a wide belt and no narrow transition zone as usually such physical geographic borderlines are.

\section{Einleitung und Fragestellung}

Wenn es Winter wird auf Hokkaido, dem $78000 \mathrm{~km}^{2}$ großen Norden Japans zwischen $41^{\circ}$ und $45^{\circ} \mathrm{NB}$, dann trägt die Insel ihren Namen zu Recht. „Hoku“ heißt Norden, „Kai“" Meer und „Dô" Land oder Straße.

Der Sommer, der wegen der von S herbeigeführten Luftmassen wärmer wird als der mitteleuropäische und den 5 Millionen Einwohnern neben dem Anbau von Kartoffeln und Mais auch die Kultivierung von Reis erlaubt, wird durch einen bitterkalten Winter kontrastiert. Die lange kalte Jahreszeit ist dafür verantwortlich, daß Hokkaido erst vor 100 Jahren japanisch-planmäßig erschlossen wurde und es heute noch in vielen Aspekten ein Pionierland darstellt. Der von $\mathrm{N}$ wehende Wintermonsun läßt das Land bereits im Oktober gefrieren. Die mittleren Januartemperaturen (Wakkanai $-5,2^{\circ} \mathrm{C}$, Asahikawa $-8,6^{\circ} \mathrm{C}$, Kitami $-9,5^{\circ} \mathrm{C}$, Obihiro $-8,1^{\circ} \mathrm{C}$ ) reichen $5-10^{\circ} \mathrm{C}$ tiefer als in Mitteleuropa. Heftige Schneefälle gehen im W und zentralen Teil der Insel nieder und bauen sich in den Tieflandsebenen zu einer über $120 \mathrm{~cm}$ dicken Schneedecke auf, die länger als 4 Monate geschlossen bleibt.

*) Anschrift der Autoren: Prof. Dr. L. E 11 e n b e r g, Institut f. Geographie, Technische Universität Berlin, Budapester Straße 44/46, D-1000 Berlin 30. - Dr. K a z u o mi H i r a k a wa, Dpt. of Geography, Yamanashi University, Kofu, Japan. 


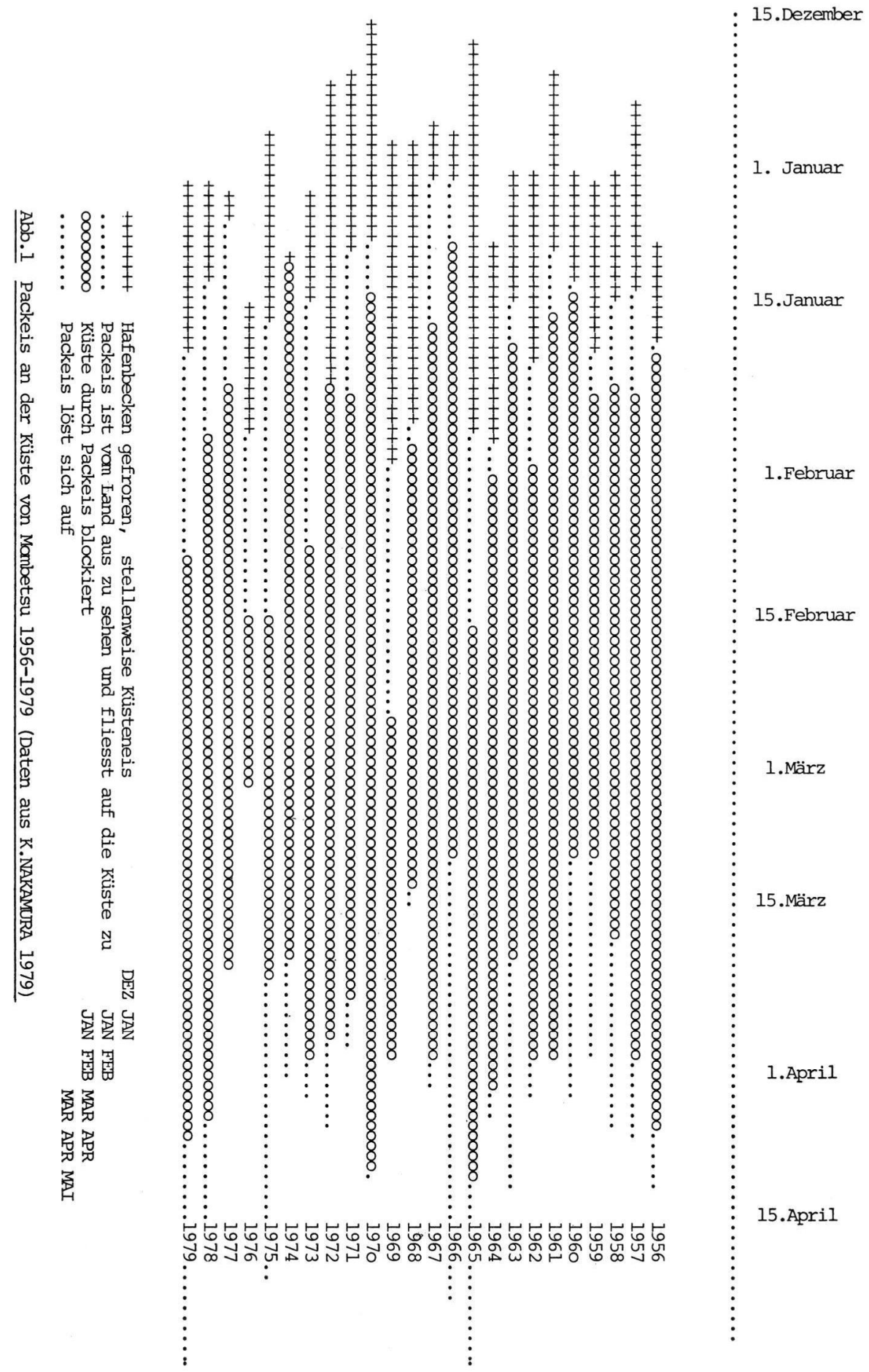


Stürme peitschen die Küste. Gischt gefriert in den Geröll- und Sandstränden. Frostsprengung setzt an den Wänden der Kliffs ein. In Hafenbecken und windgeschützten Buchten baut sich eine Eisdecke auf und im N und NE der Insel bildet sich Küsteneis. Gegen Ende des Jahres oder im Laufe des Januars sichtet man bei klarem Wetter am Horizont im $\mathrm{N}$ ein weißes Band: die Front des Treibeises. Im nördlichen Ochotskischen Meer entsteht es in jedem Winter (z. B. T. TABATA 1979), und die Strömung treibt es auf Japan zu (Abb. 1). Als Packeis riegelt es die Küsten zwischen der Nordspitze Japans und
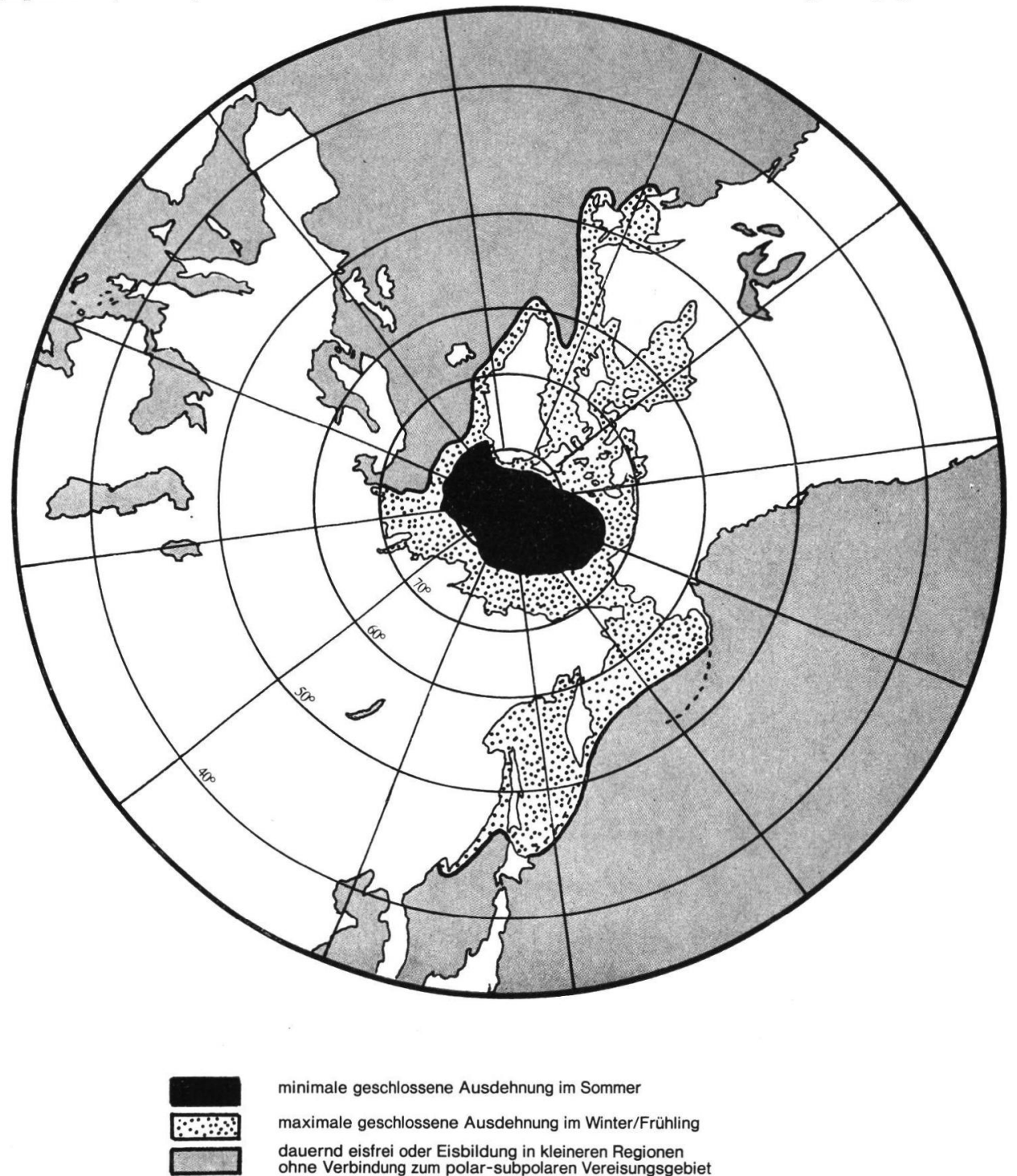

minimale geschlossene Ausdehnung im Sommer

maximale geschlossene Ausdehnung im Winter/Frühling

dauernd eisfrei oder Eisbildung in kleineren Regionen

ohne Verbindung zum polar-subpolaren Vereisungsgebiet

nach: G. Grosjean 1977

H. G. Gierloff - Emden 1980

Abb. 2: Verbreitung von Packeis. 
der Halbinsel von Nemuro allmählich ab. Der Wind drückt die Schollen ans Ufer, preßt sie an ihm entlang, schiebt sie auf die Strände oder hobelt mit ihnen an den Füßen der Kliffs. Stürme können die Eismassen zurücktreiben und neu anbranden lassen. Bis in den März hinein bleiben die Küsten eisbedrängt und der „Packeisfriedhof " bei Abashiri löst sich sogar erst im April auf.

Die Packeisküste Japans liegt zwischen $43^{\circ} 15^{\prime} \mathrm{N}$ und $45^{\circ} 31^{\prime} \mathrm{N}$. Dieser Breitenlage entspricht in Europa die Küste der Landes zwischen den Ausläufern der Pyrenäen und dem Trichter der Gironde. Nur an der Ostseite Sibiriens und Nordamerikas stößt winterliches Meereis ähnlich weit nach S vor (Abb. 2).

Die Küsten zwischen Soya Misaki und Nemuro gehören zu den äquatornächsten perimarinen Bereichen, die dem Wechsel einer wellen- und strömungsdominierten Sommerformung (8-10 Monate) und einer packeis- und frostinduzierten Winterformung (2-4 Monate) unterliegen. (Abb. 3).

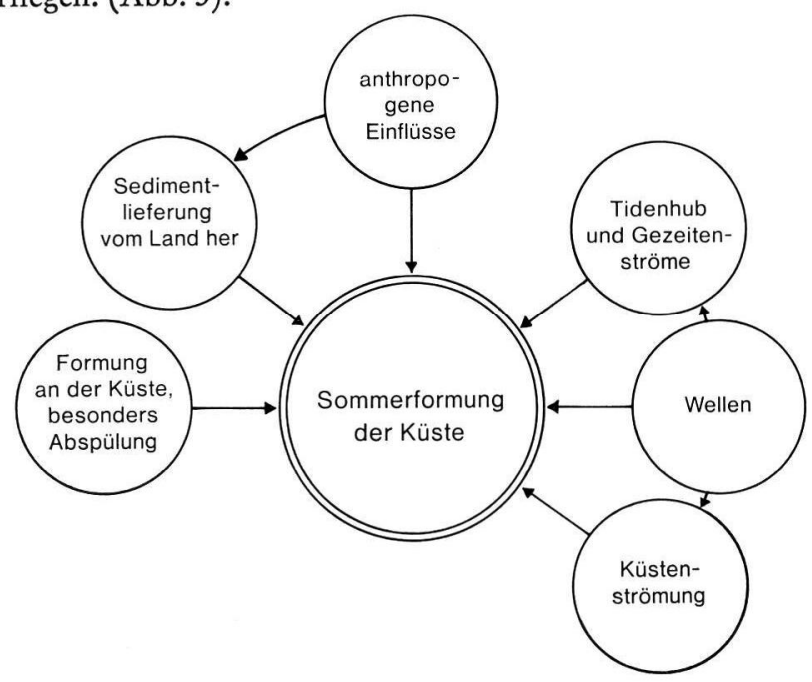

Abb. 3: Schema der Prozeßgefüge.

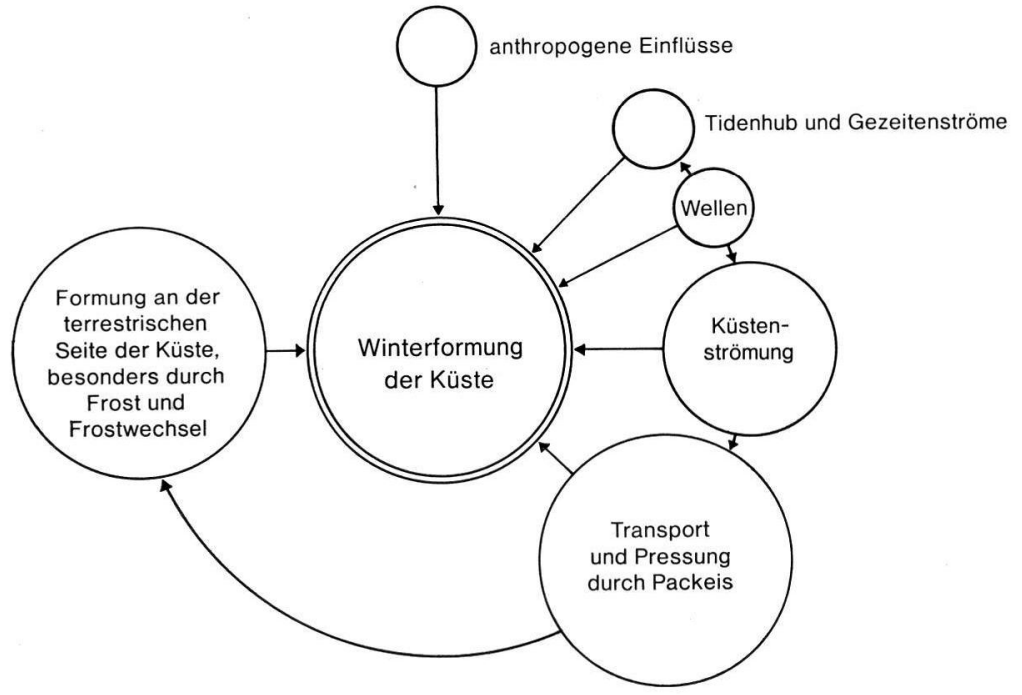


Saisonal werden bis zu 7\% der Erdoberfläche mit Eis bedeckt (B. S. JoHn \& D. E. Sudgen 1975). Für die Küsten dieses großen Raumes sind alternierend zwei derart gegensätzliche Prozeßgefüge wirksam, wie man sie sonst von keinen anderen Küsten der Erde kennt. Beobachtungen über die gegenwärtig gestaltenden Prozesse, die Entstehung von Sommer- und Winterformen an den Ufern und das Auslöschen solcher Formen sind besonders aufschlußreich, um den klima-geomorphologischen Ansatz der Küstenmorphologie zu prüfen.

Hokkaido ist durch Siedlungen, Straßen und Eisenbahnen so gut erschlossen, daß eine dreiwöchige Feldarbeit im September 1980 ausreichte, um Beobachtungen an den unterschiedlichen Küstenabschnitten durchzuführen, wobei folgende Fragen gestellt wurden:

1. Welche Prozesse sind an den verschiedenen Küsten im Sommer (und an den dauernd eisfreien Küsten ganzjährig) wirksam?

2. Wie wirkt sich die winterliche Formung auf die Küsten aus?

3. Uberdauern die saisonal gebildeten Formen in der anderen Jahreszeit?

4. Werden die Küsten stärker im Sommer oder im Winter umgestaltet?

5. Welches Ergebnis kann für eine Klimageomorphologie der Küsten der Erde gefolgert werden?

Die Autoren danken dem Sea Ice Research Laboratory der Hokkaido University für die Unterstützung in Mombetsu, der Deutschen Forschungsgemeinschaft für finanzielle Hilfe und Heidi Kunz für die Mitarbeit im Gelände.

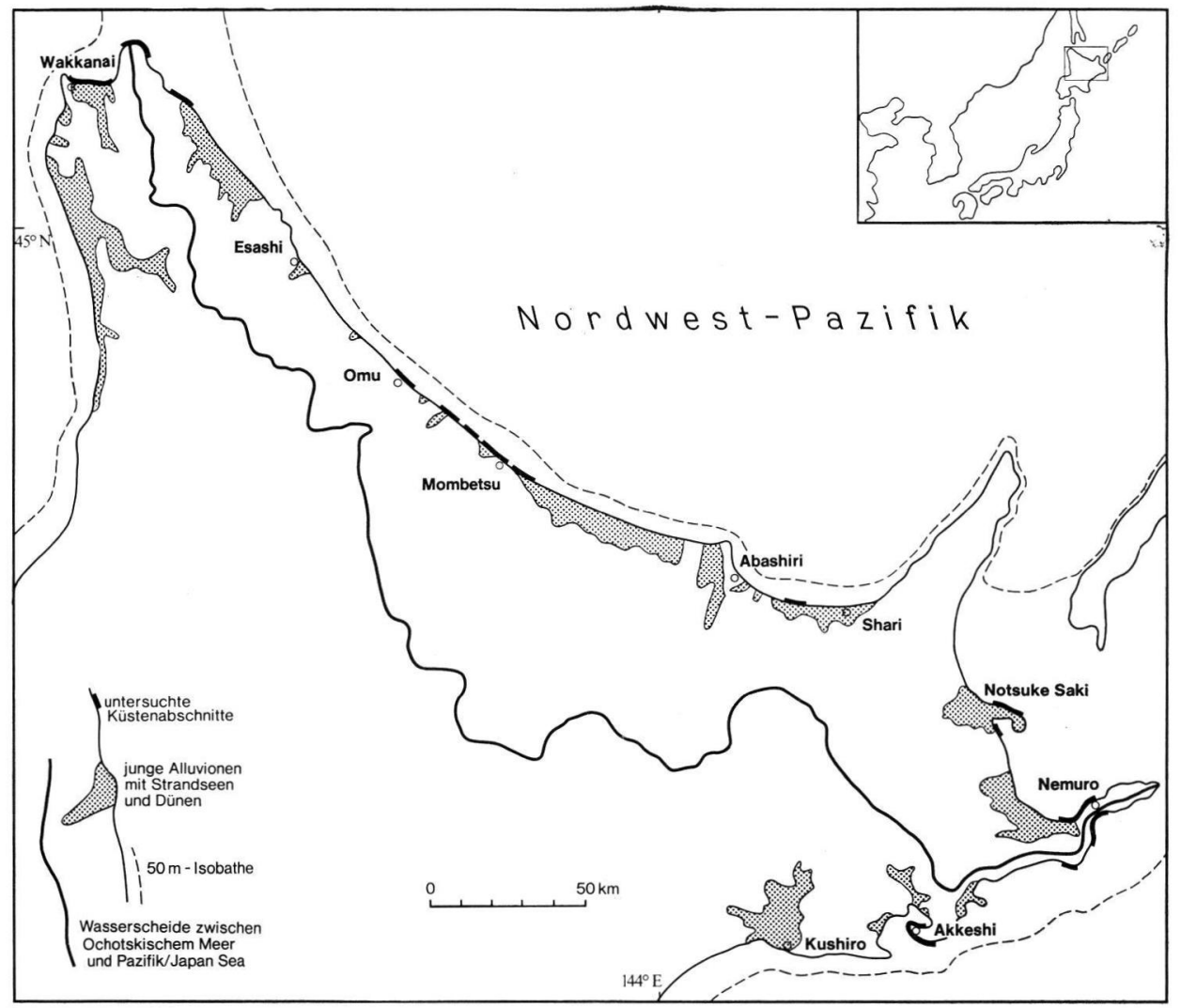

Abb. 4: Arbeitsgebiet Nordost-Hokkaido. 


\section{Arbeitsgebiet}

Das Arbeitsgebiet (Abb. 4) umfaßt die auf der Karte 1:1000 000 etwa $750 \mathrm{~km}$ messenden Küsten von Wakkanai (dauernd eisfrei) - Soya Misaki - Esashi - Omu Mombetsu - Abashiri - Shari - Nemuro (saisonal vereist) - Akkeshi - Kushiro (dauernd eisfrei).

Die geologische Vielfalt Hokkaidos spiegelt sich auch im Bereich der Packeisküste wider: paläozoische Grauwacken und Basalte, triassische und jüngere basaltdominierte Effusiva (Shiretoko Hanto), kretazische bis neogene Basalte, Siltsteine, Mergel, Sandsteine, Schiefer, Konglomerate kommen neben pleistozänen Geröllen und Sanden und holozänen Alluvionen vor.
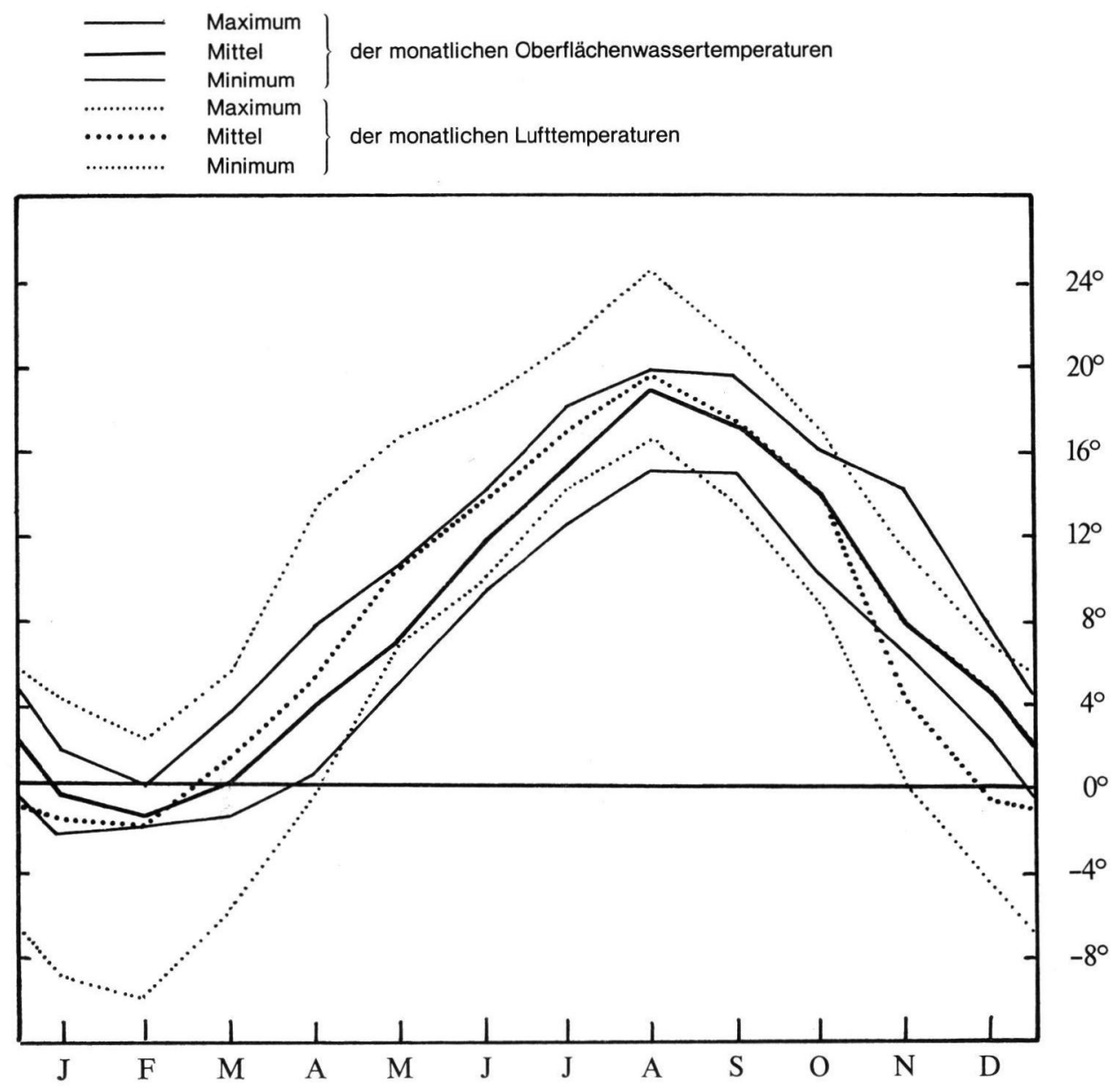

Abb. 5: Temperatur von Luft und Oberflächenwasser 1972 in Akkeshi. 
Das Küstenhinterland besteht dementsprechend aus unterschiedlichen Landformen. Entlang der Küste des Arbeitsgebietes gehören

$40 \% \mathrm{zu}$ jungen Alluvionen (marin und fluviatil) mit Strandseen, Sümpfen und Dünen,

$30 \%$ zu Terrassen und Tafeln,

$20 \%$ zu Vulkanflanken und Steilhängen,

$10 \%$ zu Hügelländern.

Lediglich im Bereich der Shiretoko Hanto, einer Vulkankette, fällt der Meeresboden steil ab und der Schelf verengt sich auf $3 \mathrm{~km}$. Im übrigen Teil des Arbeitsgebietes beträgt der Abstand von der Küste bis zur $50 \mathrm{~m}$ - Isobathe $10 \mathrm{~km}$ und mehr.

Die Höhe der Springtiden übersteigt $2 \mathrm{~m}$ im SE und schwächt sich gegen $\mathrm{N}$ ab; Watten sind nur lokal verbreitet.

Die Strömung entlang der Küsten führt generell in südliche Richtung. Sie bleibt über das ganze Jahr gleich. Kleinere Wirbel und Gegenströmungen (z. B. beim Nehrungshaken von Notsuke Saki) sind eingeschaltet. Die Geschwindigkeit bleibt während der eisfreien Zeit immer groß genug, um Sedimente entlang der Küste zu vertriften, die Mündungen der zahlreichen kurzen Flüsse zu versperren und Sandhaken weiter aufzubauen.

Die Wassertemperaturen schwanken auch im packeisfreien Bereich des Arbeitsgebietes beträchtlich. Für Akkeshi (beim Ozeanographischen Institut der Hokkaido University) sind die Jahresgänge der Oberflächenwasser- und Luftemperatur in Abb. 5 dargestellt.

Das Arbeitsgebiet stellt eine stark durchgeformte Ausgleichsküste mit starker Sedimentverdriftung dar. Besonders zwischen Soya Misaki und Shari ist deutlich zu sehen, wie Felsnasen gekappt, periglazial überformte Hügel angeklifft, Buchten verfüllt und Mündungen abgeschnürt werden.

Auch in früheren Epochen des Quartärs ähnelten die Prozeßgefüge an den Küsten den heutigen, wobei die winterliche Formung allerdings länger andauerte und auf der Landseite durch Periglazialprozesse ergänzt wurden. Gehobene Systeme küstenbegleitender Akkumulations- und Erosionsterrassen, fossile Kliffs und Strandwallserien lassen dies erkennen.

\section{Ergebnisse}

Die fünf gestellten Fragen können ganz oder teilweise beantwortet werden.

\subsection{Welche Prozesse sind an den verschiedenen Küsten im Sommer (und an den dauernd eisfreien Küsten ganzjährig) wirksam?}

An windgeschützten Fla chküsten dominieren Verlandungsprozesse. Durch Flüsse und Küstenströmungen wird das Material geliefert. An den flachen Stränden fehlen große Gerölle fast vollständig. Der Verlauf der Wasserlinie wird zusehends begradigt.

An den windexponierten Flachküsten dominieren Wellenschlag und Strandverdriftung. Auch grobes Material über $10 \mathrm{~cm}$ Durchmesser wird transportiert. Die Strände sind steiler, und oft schließt über der geglätteten Rampe normaler Wellentätigkeit eine bis zu $80 \mathrm{~m}$ breite horizontale Sandfläche - immer unbewachsen - an. Die Dynamik der Strände scheint ruckartig und der Großteil der Umformung an wenigen Sturmtagen zu erfolgen. 
Dem oberen Teil des trockenen Strandes können Windrippeln bis $30 \mathrm{~cm}$ Höhe und dünne mobile Sandschleier aufgesetzt sein. Dünen sind nicht häufig. Gut entwickeln konnten sie sich nur in den Bereichen junger Alluvionen, wo küstennahe Steilanstiege zu höher gelegenen Terrassenflächen fehlen, so z. B. bei Hamakoshimizu. Der dort $100-200 \mathrm{~m}$ breite Dünenstrang ist bis zu $30 \mathrm{~m}$ hoch, und die Formen sind höchstens zur Hälfte durch dichten Bewuchs mit Sasa stabilisiert. Daß Dünen im Sommer durch Wellenschlag angeklifft werden (Taf. 1, Fig. 1), ist an mehreren Stellen zu beobachten.

An Steilküsten wird im Sommer Grobmaterial durch Wellenarbeit zerkleinert und für den Transport aufgearbeitet. Quarrying und eventuelle Unterschneidung der Kliffs scheint sich nie unter normalen Windverhältnissen zu vollziehen, sondern nur bei Kleinkatastrophen, z. B. Taifun-Stürmen.

An allen Steilküsten, die einen schmalen vorgelagerten Strand aufweisen, bilden sich in den frostfreien Monaten, wahrscheinlich besonders rasch im Frühling, Sturzkegel aus Feinmaterial, die bis zu $4 \mathrm{~m}$ Höhe anwachsen können (Taf.1, Fig. 2). An den mergeligtonigen Steilküstenabschnitten kommen zu diesen Prozessen überdies Runsenbildung, Rutschungen und Sackungen. Die Schlipfe allein können in jedem Jahr bis zu 10\% der Hangfläche aktivieren, wie die nackten Anrisse in den sonst schütter bewachsenen Grasnarben der Kliffhänge von Ochiishi Wan zeigen.

Die sommerliche Formung ist typisch für eine Ausgleichsküste der gemäßigten Breiten mit mittleren Wind-, Tide- und Strömungsverhältnissen.

\subsection{Wie wirkt sich die winterliche Formung auf die Küsten aus?}

An der Packeisküste Japans werden während weniger Wochen bis zu vier Monaten im Winter die Brandungsprozesse ausgeschaltet und durch andere ersetzt: Transport und Pressung durch Packeis.

An mehreren Küstenabschnitten, besonders zwischen Omu und Shari, werden Eisschubkämme (ice shove ridges) aufgebaut (Sніво 1975). Bis $2 \mathrm{~m}$ über das Niveau des Springtidehochwassers werden sie strandaufwärts geschoben. Sie bestehen aus Sand lokaler Herkunft und aus Eis. Die $50 \mathrm{~cm}$ bis $2 \mathrm{~m}$ hohen Wälle sind zunächst prägnante Formen (NAKAmura 1979), sacken beim Schmelzen im März/April etwas in sich zusammen und werden von den Wellen angeklifft (Taf. 1, Fig. 3 und 4). Ice shove ridges aus Kies sind seltener, kommen aber vor und unterscheiden sich von Strandwällen durch ein unregelmäßigeres Kleinrelief. Nirgends werden die Wälle über den trockenen Strand ganz hinausgeschoben.

Pressung durch Packeis kann auch wiederholt erfolgen, wenn Stürme die Eisfront zurückdrängen, was an der Küste westlich Nemuro bis zu fünf mal pro Winter vorkommen soll (Mitt. von Fischern). Block- und Geröllpackungen am Fuß von Kliffs können so in Nischen hineingeschoben und zu $150 \mathrm{~cm}$ hohen Terrassenkörpern aufgepreßt werden.

An der Meerseite der meisten Küstendünen, so auch bei Hamakoshimizu, fallen ein oder zwei Verflachungen auf (Taf. 1. Fig. 5). Sie liegen über dem Bereich des Wellenschlages. Sie sind schütterer bewachsen als die übrigen Teile der Dünen. Die Terrassenkante begrenzt die Horizontalfläche deutlich, was das Relief, und messerscharf, was den Bewuchs anbetrifft. Pressung der Dünen und Überschleifen mit Eis können für die Entstehung dieser Formen in Frage kommen.

Die sandigen Akkumulationen der Flachküsten gefrieren im Winter. Transport und Pressung durch Packeis sind dort die einzigen winterlichen Prozesse, die zu einer Formengestaltung führen. Anders ist es an Kliffs, wo Regenwasser und Gischt in die Gesteins- 

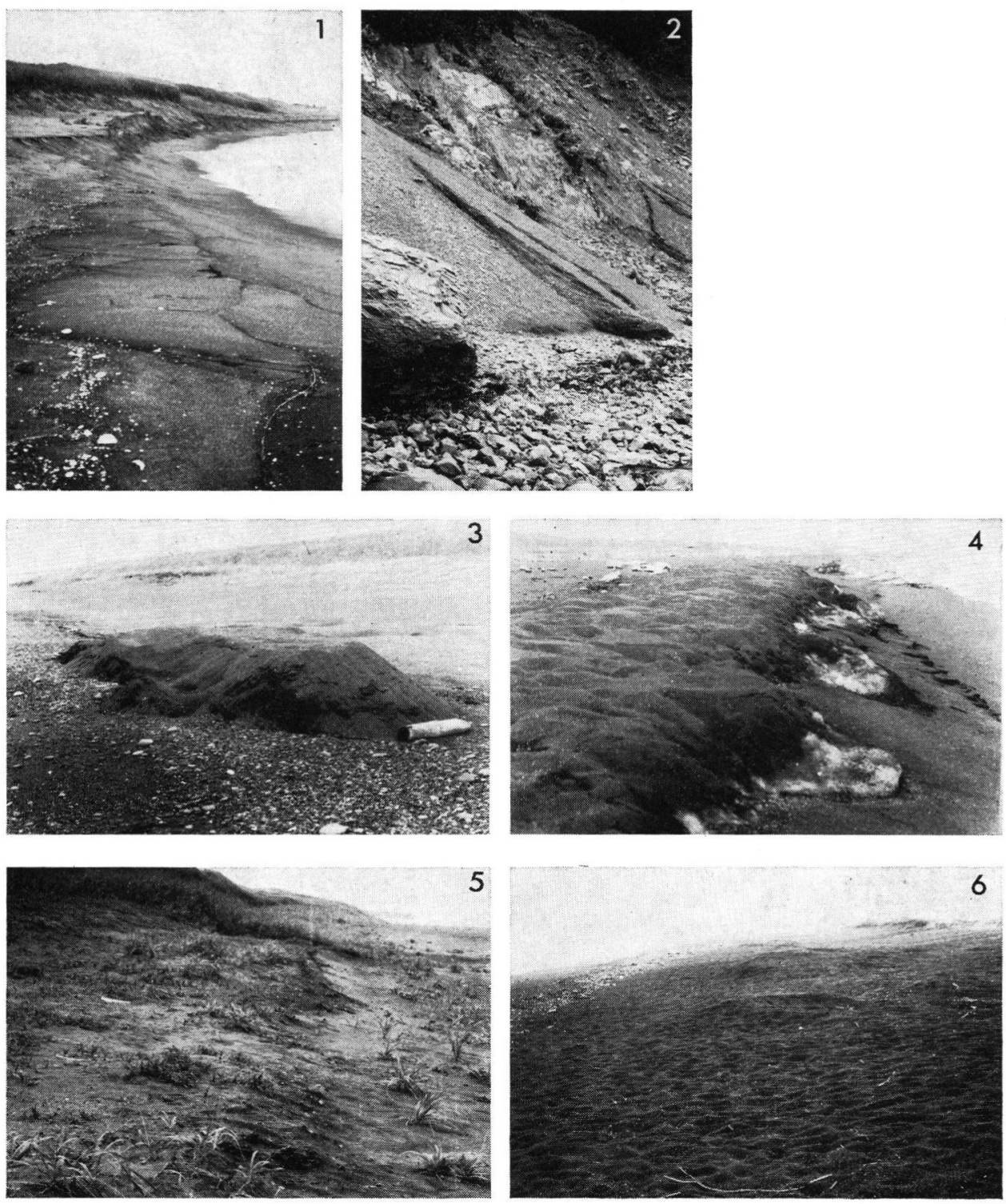

\section{T a f e l I}

Fig. 1: Angekliffte Düne bei Hamakoshimizu (Ellenberg, Sept. 1980).

Fig. 2: Sommerlich geformter Sturzkegel aus Feinmaterial bei Akkeshi (Ellenberg, Sept. 1980) Fig. 3: Ice shove ridge bei Mombetsu (Nakamura, Apr. 1978)

Fig. 4: Angekliffter ice shove ridge bei Mombetsu (Nakamura, Apr. 1978)

Fig. 5: Verflachungen in den Dünen von Hamakoshimizu (Ellenberg, Sept. 1980)

Fig. 6: Rest eines ice shove ridges im späten Sommer bei Mombetsu (EllenberG, Sept. 1980, Gleiche Stelle wie Fig. 3). 
fugen - besonders bei basaltigem Material - eindringen, gefrieren und zu Frostsprengung führen. Halden mit kaum kantengerundeten großen Blöcken an den Füßen der Kliffs und frische unverwitterte Gesteinspartien an den Wänden zeugen von dieser Dynamik. Viele der Kliffs zeigen wegen dieser rasch voranschreitenden frostdynamischen Zerlegung der Wand kaum Unterschneidungen und Hohlkehlen, aber einen Blockschuttmantel, der sich nach Aufarbeitung und Abtransport schnell erneuert.

\section{3. Überdauern die saisonal gebildeten Formen in der anderen Jahreszeit?}

Die im Som mer entstandenen oder weiter geformten und umgestalteten Verlandungssäume, Strände, Dünen, Runsen und Rutschungen überdauern den Winter ohne große Veränderung. Die weitere Umgestaltung dieser Formen setzt nach dem Abschmelzen des Eises dort ein, wo sie im Dezember oder Januar aufgehört hat.

Eine Ausnahme bilden die Feinerde-Sturzkegel, die in sturmfreien Perioden entstehen und jedes oder fast jedes Jahr durch einmalige Ereignisse starken Wellenschlages oder Eisschubs (also Sommer- oder Winterformung) beseitigt oder verletzt werden.

Die im W in t e r aufgeschobenen ice shove ridges werden zunächst durch den Schmelzvorgang verändert. Sie sacken in sich zusammen, und aus scharfen Kammformen werden $2 \mathrm{~m}$ breite und $50 \mathrm{~cm}$ hohe Rücken mit unregelmäßigem Kleinrelief (NAKAMURA 1979). Schon im Frühling kann eine Kliffung beginnen. Wellen und auch Wind gestalten die Formen derart stark um, daß sie im späten Sommer nur noch andeutungsweise zu erkennen sind (Taf.1, Fig. 6). An vielen Stellen bleiben die ice shove ridges auch nur einige Monate erhalten und werden dann ganz beseitigt.

Die Block- und Geröllpackungen (eisgepreßt oder als Halden) und die Verflachungen in Dünen sind Formen, die längerfristig überdauern.

\subsection{Werden die Küsten stärker im Sommer oder im Winter umgestaltet?}

An Flachk üsten ist der Sommer die morphodynamisch ausschlaggebende Zeit. Winter heißt hier eher Formungsruhe oder Dekoration mit vergänglichen Formen als bleibende Umgestaltung. Da die eisfreie Zeit $2 / 3$ bis $11 / 12$ des Jahres ausmacht, sollte dieser Befund nicht überraschen.

An $\mathrm{Steilküsten} \mathrm{mit} \mathrm{Kliffs} \mathrm{ist} \mathrm{diese} \mathrm{Frage} \mathrm{nicht} \mathrm{einfach} \mathrm{zu} \mathrm{beantworten.} \mathrm{Quar-}$ rying und Abtransport durch Wellen im Sommer und Wandzerlegung und Blockzerkleinerung durch Frost/Frostwechsel im Winter und Frühjahr sind eine Prozeßkombination, die sich morphodynamisch ergänzt. Die Formung durch Packeis ist an den meisten Steilküstenabschnitten nicht auffallend.

So ist die Packeisküste Japans keine Küste, die in erster Linie während der Wintermonate ihr Gepräge erhält, sondern ein perimariner Raum mit intensiver Sommer- und schwacher Winterformung im Übergang vom gemäßigten zum subpolaren Formungsstil.

\subsection{Welches Ergebnis kann für eine Klimageomorphologie der Küsten der Erde gefolgert werden?}

Die Nord- und Nordostküste Hokkaidos gehört zwar zu den von Treibeis erreichten Küsten der Erde, stellt aber gegenüber den Packeisküsten des nördlichen Eurasiens (z. B. Reinhard 1958/59, Ionin et al. 1961, Zenkovich 1967) oder des subpolaren Nordamerikas (z. B. Nichols 1953, John \& Sugden 1975) und Ostkanadas (Dionne 1972, 1980) eine Ausnahme dar, da die Winterformen sehr vergänglich und nur schwach ausgebildet sind. 
Aus Arbeiten über andere Packeisküsten bekannte litorale Furchen und Mulden, Eisschubkämme aus Blöcken bis $5 \mathrm{~m}$ Höhe, Druckmoränen mit tonnenschweren Geschieben und Sedimentpackungen, die mehr als $500 \mathrm{~m}$ landeinwärts geschoben werden, gibt es in Japan nicht.

Drei Gründe sind dafür zu nennen, daß die japanische Küste eine nur schwache Prägung durch Packeis erfährt:

1. Die Packeisformung dauert nur einige Wochen oder wenige Monate.

2. Zuvor gebildetes Küsteneis kann die Formen erstarren und Treibeis-Pressung verhindern lassen.

3. Den Küsten sind keine aufzupflügende Watten vorgelagert.

Bei einer Betrachtung der Küsten der Erde aus klimageomorphologischer Sicht (DAviEs 1972; Valentin 1979; Ellenberg 1980) ist eine Grenze zwischen tropischen und gemäßigten Küsten relativ leicht zu ziehen (z. B. als $18^{\circ} \mathrm{C}$-Isotherme des Oberflächenwassers im kältesten Monat). Die Grenze zwischen einer gemäßigten und subpolaren Zone bzw. einer intermediären und einer kühlen (ELLENBERG, im Druck), ist dagegen schwieriger festzulegen. Sie mit der winterlichen Verbreitungsgrenze von geschlossenem Treibeisvorkommen gleichzusetzen ist ein immerhin sinnvoller Vorschlag, da gegenüber wärmeren Küstenregionen andersartige Prozesse die Morphodynamik ergänzen und ein Wechsel von Sommer- und Winterformung geomorphologisch bestimmend wird.

Diese Grenze ist ein breiter Saum und kein schmaler Übergang, wie es für solche physisch geographischen Grenzen in vielen Bereichen der Fall ist.

\section{Schriftenverzeichnis}

Davies, J. L. (1972): Geographical variations in coastal development (Geomorphology Texts 4) 204 pp., Edinburgh.

Dionne, J. C. (1972): Caractéristiques des schorres des régions froides, en particulier de l'éstuaire du Saint-Laurant. - Z. Geomorph. N. F. Suppl. Bd. 13: 131-162; Stuttgart (Bornträger).

- (1980): Les glaces comme agent littoral sur la côte orientale de la Baie des James Québec. Proc. Canadian Coastal Conference 1980: 80—92; Burlington, Ontario.

Ellenderg, L. (1980): Zur Klimamorphologie tropischer Küsten. - Berliner Geogr. Studien, 7: 177-192; Berlin.

- (1982): Climatic morphological aspects of world's coasts. - Erdkunde; Bonn. — [Im Druck.]

Gierloff-Emden, H. G. (1980): Geographie des Meeres, 1. Teil. - 766 pp.; Verlin - New York (Gruyter).

Grosjean, G. (1977): Die Erde. Natur, Mensch, Wirtschaft. - Wandkarte. - Bern (Kümmerly und Frey).

Ionin, A. S. et al. (1961): Einige Resultate regionaler Forschungen an den Meeresküsten der USSR. Aus: AN SSR, Trudy instituta okeanologii, 48. - [Unveröff. Übersetzung v. G. KRäMER).

John, B. S. \& D. E. Sudgen (1975): Coastal geomorphology of high latitudes. - Progress in Geography, 7: 53-132; London.

Nakamura, K. (1979): A transformation of sea ice and coastal land-form on the coast of the Sea of Okhotsk in Winter. - Bull. Dohto Univ., Fac. Fine Arts, 1979/2: 68-95; Mombetsu. [Japanisch.]

Nichols, R. L. (1953): Marine and lacustrine ice-pushed ridges. - J. Glaciol., 2 (13): 172-175; Cambridge.

Reinhard, H. (1958/59): Über Wirkungen des Eises an der Küste. - Wiss. Z. Univ. Greifswald, 8, Math.-Nat. Wiss. Reihe 1/2: 135-141; Greifswald. 
SHibor, T. (1975): The sorted polygons at Hirayama and the ice-shove ridges along the beach of Ochotsk Sea. - J. Geography, Tokyo University, 84: 41-52; Tokyo.

TABAta, T. et al. (1979): Distribution of pack ice of Ochotsk Sea Coast of Hokkaido observed with sea ice radar network January - May 1979. - Low Temperature Science, A 38: 39-70; Hokkaido University, Sapporo. - [Japanisch].

Valentin, H. (1979): Ein System der zonalen Küstenmorphologie. - Z. Geomorph. N. F., 23 (2): 113-131; Stuttgart.

Zenkovich, V. P. (1967): Processes of coastal development. - Edinburgh/London. - [aus dem Russischen übersetzt von D. G. Fry.]

Manuskript eingegangen am 6. 11. 1981. 